PROCEEDINGS OF THE

AMERICAN MATHEMATICAL SOCIETY

Volume 128, Number 3, Pages 845-852

S 0002-9939(99)05065-0

Article electronically published on July 28, 1999

\title{
SPECTRAL CHARACTERIZATION OF SOLUTIONS TO SYSTEMS OF LINEAR DIFFERENTIAL EQUATIONS
}

\author{
J. W. NEUBERGER
}

(Communicated by Christopher D. Sogge)

\begin{abstract}
A spectral characterization of solutions of abstract linear differential equation systems is given. The characterization is in terms of the spectrum of a related continuous self-adjoint linear operator.
\end{abstract}

\section{INTRODUCTION}

Some systems of partial differential equations have boundary conditions under which there is one and only one solution. Then there are disturbing equations (Burgers' equation with zero viscosity, the Tricomi equation, ...) for which adequate boundary conditions are not known. For Burgers' equation (with zero viscosity) on a square disk, for example, given an interval on the boundary, there may be placed smooth boundary conditions for which there is no solution. For Tricomi's equation there are some special regions for which this equation is somewhat understood (see [5] for a recent discussion) in terms of boundary conditions, but for even a rectangle the situation is far from clear.

For a given system of partial differential equations on a given region, one might want a characterization of the set of all solutions even though a characterization in terms of boundary conditions is not available. From such a characterization, one might be able to extract a particular solution fitting ones requirements.

In this note we give a spectral characterization of the set of all solutions for a rather general class of linear systems. To each system of the type studied we associate a bounded self-adjoint linear transformation. The set of all solutions to a system is characterized in terms of the spectrum of this associated transformation.

\section{Abstract Setting}

Denote by each of $H, K$ and $J$ a Hilbert space and denote by $T$ a closed densely defined linear transformation from $H$ to $K$. Denote by $H_{T}$ the Hilbert space whose points consist of $d m(T)$ (the domain of $T$ ) where

$$
\|x\|_{H_{T}}=\left(\|x\|_{H}^{2}+\|T x\|_{K}^{2}\right)^{1 / 2}, x \in H_{T},
$$

Received by the editors March 5, 1998 and, in revised form, May 5, 1998.

1991 Mathematics Subject Classification. Primary 35A35.

(C)1999 American Mathematical Society 
the graph norm of $x$ with respect to the transformation $T$. Define $D: d m(T) \rightarrow$ $H \times K$ by

$$
D x=\left(\begin{array}{c}
x \\
T x
\end{array}\right), x \in H_{T} .
$$

Suppose also that $B \in L(H, J), C \in L(K, J)$ and $A \in L(H \times K, J)$ so that

$$
A\left(\begin{array}{l}
x \\
y
\end{array}\right)=B x+C y,\left(\begin{array}{l}
x \\
y
\end{array}\right) \in H \times K .
$$

Note that $A^{t}: J \rightarrow H \times K$ is given by

$$
A^{t} w=\left(\begin{array}{l}
B^{t} w \\
C^{t} w
\end{array}\right), w \in J
$$

In this note we are mainly concerned with the problem of determining $u \in H_{T}$ so that

$$
A D u=0 .
$$

Clearly a vast collection of linear homogeneous systems are represented by this general problem.

Define $G: H_{T} \rightarrow J$ by

$$
G=A D .
$$

We now specify $G^{*} \in L\left(J, H_{T}\right)$, the Hilbert space adjoint of $G$.

Denote by $P$ the orthogonal projection of $H \times K$ onto $R(D)$, the range of $D$ (i.e., the graph of $T$ ). Denote by $\pi: H \times K \rightarrow H$ the transformation such that

$$
\pi\left(\begin{array}{l}
x \\
y
\end{array}\right)=x,\left(\begin{array}{l}
x \\
y
\end{array}\right) \in H \times K
$$

Lemma 1. $G^{*}=\pi P A^{t}$.

Proof. If $x \in H_{T}, h \in J$,

$$
\begin{aligned}
\langle G x, h\rangle_{J}=\langle A D x, h\rangle_{J} & =\left\langle D x, A^{t} h\right\rangle_{H \times K} \\
& =\left\langle P D x, A^{t} h\right\rangle_{H \times K}=\left\langle D x, P A^{t} h\right\rangle_{H \times K}=\left\langle x, \pi P A^{t} h\right\rangle_{H_{T}}
\end{aligned}
$$

so $G^{*}=\pi P A^{t}$.

In what follows we use the symbol $j$ to denote the identity function on $R$, i.e., $j(t)=t, t \in R$. This will help keep our notation shorter and we hope clearer.

Denote $A P A^{t}=G G^{*}$ by $Q$ and denote by $\phi$ a spectral family for $Q$ :

$$
Q=\int_{0}^{b} j d \phi
$$

where $b=\sup _{x \in J,\|x\|_{J}=1}\langle Q x, x\rangle_{J}$. Then $[0, b]$ contains the numerical range of $Q$ (see [4] for a general reference on spectral theory, numerical range, graph norm). Denote by $\gamma:[0, b] \rightarrow L(J, J)$ the function so that

$$
\gamma(\lambda)=G^{*} \phi(\lambda) G, \lambda \in[0, b] .
$$

Denote by $\Gamma$ the orthogonal projection of $H_{T}$ onto $N(A D)$.

\section{Theorem 1.}

$$
\Gamma u=u-\lim _{a \rightarrow 0+} \int_{a}^{b} 1 / j d \gamma u, u \in N(A D) \oplus R\left(\pi P A^{t}\right) .
$$


Since the $H_{T}$ closure of $R\left(\pi P A^{t}\right)=R\left((A D)^{*}\right)$ is the $H_{T}$ orthogonal complement of $N(A D)$, it follows that $N(A D) \oplus R\left(\pi P A^{t}\right)$ is dense in $H_{T}$. A proof of the theorem is given later in this note. We remark that if $Q$ is compact, then the statement of the theorem has the simpler form of an eigenvector expansion.

\section{A simple example}

We specialize the various spaces and transformations in the preceding section to the case of the problem of determining all $y$ on $[0,1]$ so that

$$
y^{\prime}-y=0 \text { on }[0,1] .
$$

We hope that this concrete example helps fix the ideas of the preceding section.

Take $H=K=J=L_{2}([0,1]), T f=f^{\prime}, f \in H^{[1,2]}([0,1]), B=-I$ on $H, C=$ $I$ on $K$. Take

$$
A\left(\begin{array}{l}
f \\
g
\end{array}\right)=B f+C g=-f+g,\left(\begin{array}{l}
f \\
g
\end{array}\right) \in H \times K .
$$

Then

$$
A^{t} h=\left(\begin{array}{c}
-h \\
h
\end{array}\right), h \in J
$$

Thus $H_{T}$ is the Sobolev space $H^{(1,2)}([0,1])$ and $D u=\left(\begin{array}{c}u \\ u^{\prime}\end{array}\right), u \in H_{T}$ ([1] is our general reference for Sobolev spaces). It remains to indicate the nature of the projection $P$ in this special case. To this end observe that the orthogonal complement in $H \times K$ of

$$
\left\{\left(\begin{array}{c}
u \\
u^{\prime}
\end{array}\right): u \in H_{T}\right\}
$$

is

$$
\left\{\left(\begin{array}{c}
v^{\prime} \\
v
\end{array}\right): v \in H_{T}, v(0)=0=v(1)\right\} .
$$

Accordingly, if $\left(\begin{array}{l}f \\ g\end{array}\right) \in H \times K$, there exist unique $u, v \in H_{T}$ so that

$$
\left(\begin{array}{c}
u \\
u^{\prime}
\end{array}\right)+\left(\begin{array}{l}
v^{\prime} \\
v
\end{array}\right)=\left(\begin{array}{l}
f \\
g
\end{array}\right), v(0)=0=v(1)
$$

It is an excercise in elementary ordinary differential equations to see that (we use $C(x)=\cosh x, S(x)=\sinh x, x \in R)$

$$
\begin{aligned}
& \text { (3.1) } u(x)= \\
& \quad\left(C(1-x) \int_{0}^{x}(C f+S g)+C(x) \int_{x}^{1}(C(1-j) f-S(1-j) g)\right) / S(1), x \in[0,1] .
\end{aligned}
$$

Further straightforward calculation yields that

$$
A P A^{t} w=w+\langle w, q\rangle_{J} q, w \in J
$$

where

$$
q(x)=e^{-x} \sqrt{e / S(1)}, x \in R\left(\|q\|_{L_{2}([0,1])}=1\right) .
$$

Note that the spectrum of $Q$ consists of $\{1,2\}$ with $q$ an eigenfunction for $Q$ with eigenvalue 2 and any non-zero member of $L_{2}([0,1])$ which is $L_{2}$ orthogonal to $q$ an eigenfunction for $Q$ with eigenvalue 1 . 
Interpreting Theorem 1 for this example we have

$$
\begin{aligned}
& \int_{0}^{b} 1 / j d \gamma u= \lim _{a \rightarrow 0+} \int_{a}^{b} 1 / j d \gamma u \\
&=\pi P A^{t}\left[\left(A D u-\langle A D u, q\rangle_{H} q\right)+(1 / 2)\langle A D u, q\rangle_{H} q\right] \\
&=\pi P A^{t}\left[A D u-(1 / 2)\langle A D u, q\rangle_{H} q\right] \\
& \quad=\pi P A^{t}\left(u^{\prime}-u\right)-(1 / 2)\left\langle\left(u^{\prime}-u\right), q\right\rangle_{H} \pi P A^{t} q .
\end{aligned}
$$

Now $\pi P A^{t} q=\pi P\left(\begin{array}{c}-q \\ q\end{array}\right)=-q$ since $q^{\prime}=-q$. Moreover

$$
\left\langle u^{\prime}-u, q\right\rangle_{H}=\int_{0}^{1}(u q)^{\prime}=u(1) q(1)-u(0) q(0) .
$$

Hence

$$
(1 / 2)\left\langle u^{\prime}-u, q\right\rangle_{H}\left(\pi P A^{t} q\right)(x)=-(e / 2 S(1))\left(u(1) e^{-1}-u(0)\right) e^{-x}, x \in[0,1] .
$$

On the other hand,

$$
\pi P A^{t}\left(u^{\prime}-u\right)=\pi P\left(\begin{array}{c}
u \\
u^{\prime}
\end{array}\right)-\pi P\left(\begin{array}{c}
u^{\prime} \\
u
\end{array}\right)=u-\pi P\left(\begin{array}{l}
u^{\prime} \\
u
\end{array}\right) .
$$

We want to evaluate $\pi P\left(\begin{array}{l}u^{\prime} \\ u\end{array}\right)$. By (3.1) after some simplification,

$$
\pi P\left(\begin{array}{l}
u^{\prime} \\
u
\end{array}\right)(x)=-(C(1-x) u(0)-C(x) u(1)) / S(1), x \in[0,1],
$$

and hence after more simplification,

$$
\int_{0}^{b} 1 / j d \gamma u=u-\left(u(1)-u(0) e^{-1}\right) e^{j} /(2 S(1))
$$

and so

$$
u-\int_{0}^{b} 1 / j d \gamma u=(1 /(2 S(1)))\left(u(1)-u(0) e^{-1}\right) e^{j} .
$$

The reader may check that this last expression is the nearest element of $N(A D)$ to $u$ (in the $H_{T}$ metric).

\section{Discussion}

In cases such as the above example in which $N(A D)$ is known explicitly, the orthogonal projection onto $N(A D)$ can be calculated directly. Intended applications are to problems such as the Tricomi equation in Section 5 in which $N(A D)$ is not known explicitly.

As an alternative we could regard $A D$ as a closed unbounded operator $L$ on a dense subset $D(T)$ of $H$ (think of $L$ as a linear differential operator). Then $L^{t} L$ is a self-adjoint operator of order twice that of $L$ itself. How might a spectral analysis of $L^{t} L$ be made as an alternative to our present analysis? First, there is the problem of coming to terms with $L^{t}$, both its form and its domain. The operator $L$ may already have associated boundary conditions implicit in the choice of $T$. For instance, in the above example one might have had

$$
T f=f^{\prime}, f \in H^{1,2}([0,1]), f(0)=0,
$$


with the domain of $T$ precisely such functions $f$. This would change the projection $P$ and consequently $A P A^{t}$ even though $A$ remains the same. Such boundary conditions might be quite complicated as indicated in Chapters 5 and 6 of [3]. The domain of $L^{t}$ would generally involve boundary conditions which have to be determined by an integration by parts analysis. Once this problem is overcome, one would still be faced with a spectral analysis of the unbounded operator $L^{t} L$. These difficulties might be compared with the problem of a spectral analysis of the bounded self-adjoint operator $A P A^{t}$.

In such a spectral analysis of $A P A^{t}$ in applications, often $A$ is of rather simple nature. Since so much of our theory depends on $P$, some discussion of its nature seems in order. For $T$ a closed densely defined linear transformation on $H$ to $K$, the orthogonal projection of $H \times K$ onto the range of the associated transformation $D$ (the graph of $T$ ) is given by a formula of von Neumann [6]:

$$
P\left(\begin{array}{l}
x \\
y
\end{array}\right)=\left(\begin{array}{c}
\left(I+T^{t} T\right)^{-1} x+T\left(I+T T^{t}\right)^{-1} y \\
T\left(I+T^{t} T\right)^{-1} x+y-\left(I+T T^{t}\right)^{-1} y
\end{array}\right),(x, y) \in H \times K .
$$

The transformations $\left(I+T^{t} T\right)^{-1},\left(I+T T^{t}\right)^{-1}$ are discussed in Chapter VIII of [4]. They are inverses of laplacians associated with the Dirichlet spaces $\left(H, H_{T}\right)$ and $\left(K, K_{-T^{t}}\right)$ respectively (see Beurling-Deny [2], 191-208, or [3], Chapter 5).

We remark that in our general setting, $N\left(\pi P A^{t}\right)$ is the orthogonal complement (in the metric $H_{T}$ ) of $R(A D)$. Hence zero is an eigenvalue of $A P A^{t}$ if and only if $A D$ does not have range dense in the space $J$. Thus a full spectral analysis of $A P A^{t}$ carries information about $R(A D)$ as well as about $N(A D)$.

\section{TRICOMI'S EQUATION}

Denote by $\Omega$ a bounded region in $R^{2}$. Tricomi's equation on $\Omega$ is the problem of finding $u$ on $\Omega$ such that

$$
y u_{1,1}(x, y)+u_{2,2}(x, y)=0,(x, y) \in \Omega .
$$

Letting $j_{2}: R^{2} \rightarrow R$ denote the function such that $j_{2}(x, y)=y,(x, y) \in R^{2}$, we rewrite (5.1) as

$$
j_{2} u_{1,1}+u_{2,2}=0 \text {. }
$$

We try to fit this problem into the setting of Section 2. Define $H=J=L_{2}(\Omega), K=$ $L_{2}(\Omega)^{2}$,

$$
T u=\left(\begin{array}{l}
u_{1,1} \\
u_{2,2}
\end{array}\right), u \in H^{2,2}(\Omega) .
$$

Define $B u=0, u \in H ; C\left(\begin{array}{l}r \\ s\end{array}\right)=j_{2} r+s ; A\left(\begin{array}{l}u \\ \left(\begin{array}{l}r \\ s\end{array}\right)\end{array}\right)=C\left(\begin{array}{l}r \\ s\end{array}\right), u \in H,\left(\begin{array}{l}r \\ s\end{array}\right) \in K . H_{T}$ then becomes the space whose points are those of $H^{2,2}(\Omega)$ with

$$
\|u\|_{H_{T}}=\left(\int_{\Omega}\left(u^{2}+u_{1,1}^{2}+u_{2,2}^{2}\right)\right)^{1 / 2}, u \in H_{T} .
$$

What we want is a full spectral analysis of $A P A^{t}$ in this case. Right now this is beyond this writer's ability but we do remark that following [3] one can approximate $A P A^{t}$ by finite dimensional versions. For such finite dimensional versions the associated spectral problems are accesible numerically. Maybe some numerical experimentation would shed light on spectral properties of $A P A^{t}$. 


\section{Continuous steepest Descent}

The orthogonal projection in Theorem 1 and its complementary projection are both characterized by means of continuous steepest descent. In this section we assume the notation of Section 2.

First take

$$
\alpha(u)=\|A D u\|_{J}^{2} / 2, u \in H_{T} .
$$

Then

$$
\alpha^{\prime}(u) h=\langle A D h, A D u\rangle_{J}=\left\langle h, \pi P A^{t} A D u\right\rangle_{H_{T}}
$$

and hence

$$
(\nabla \alpha)(u)=\pi P A^{t} A D u, u \in H_{T} .
$$

In [3], Chapter 3, it is shown that if

$$
z(0)=u_{0} \in H_{T}, z^{\prime}(t)=-(\nabla \alpha)(z(t)), t \geq 0,
$$

then

$$
u=\lim _{t \rightarrow \infty} z(t) \text { exists and } A D u=0
$$

Moreover, $u$ is the nearest solution in $H_{T}$ to $u_{0}$ in the $H_{T}$ norm.

Now fix $u \in H_{T}$ and define

$$
\beta(v)=\left\|\pi P A^{t} v-u\right\|_{H_{T}}^{2} / 2, v \in H_{T} .
$$

Then

$$
\beta^{\prime}(v) h=\left\langle\pi P A^{t} h, \pi P A^{t} v-u\right\rangle_{H_{T}}=\left\langle h, A P A^{t} v-A D u\right\rangle_{J}, v, h \in H_{T} .
$$

Hence

$$
(\nabla \beta)(v)=A P A^{t} v-A D u, v \in H_{T} .
$$

Theorem 2 provided a motivation for Theorem 1 . In case $A P A^{t}$ has an inverse which is in $L(J, J)$, it was noticed that Theorem 2 leads, through a spectral representation of $A P A^{t}$, to the truth of Theorem 1. It was then established that the formula of Theorem 1 holds anyway.

Theorem 2. Suppose $z(0)=v_{0} \in J$ and $z^{\prime}(t)=-(\nabla \beta)(z(t)), t \geq 0$. Then

$$
w=\lim _{t \rightarrow \infty} \pi P A^{t} z(t) \text { exists }
$$

and

$$
\|w-u\|_{H_{T}}<\|g-u\|_{H_{T}} \text { for all } g \in \text { the } H_{T} \text { closure of } R\left(\pi P A^{t}\right), g \neq w \text {. }
$$

We will see that $w$ is the orthogonal projection of $u$ onto the $H_{T}$ closure of $R\left(\pi P A^{t}\right)$. A proof of this result is in the next section. 


\section{SOME LEMMAS AND PROOFS}

Lemma 2. Suppose $v \in J$ and $w=\pi P A^{t} v$. Then

$$
\lim _{a \rightarrow 0+} \int_{a}^{b} 1 / j d \gamma w=w
$$

Proof. If $0<a<b$, then

$$
\begin{aligned}
\int_{a}^{b} 1 / j d \gamma w= & \pi P A^{t}\left(\int_{a}^{b} 1 / j d \phi\right) A D w \\
& =\left(\pi P A^{t}\right)\left(A P A^{t}\right) \int_{a}^{b} 1 / j d \phi v=\pi P A^{t} \int_{a}^{b} d \phi v
\end{aligned}
$$

and so

$$
\int_{a}^{b} 1 / j d \gamma w=\pi P A^{t}(\phi(b)-\phi(a)) v
$$

and hence

$$
\lim _{a \rightarrow 0+} \int_{a}^{b} 1 / j d \gamma w=\pi P A^{t} v=w
$$

since $\lim _{a \rightarrow 0+} \phi(a) v$ is the orthogonal projection of $J$ onto $N\left(A P A^{t}\right)=N\left(\pi P A^{t}\right)$.

Define $E_{0}$ so that

$$
E_{0} u=\lim _{a \rightarrow 0+} \int_{a}^{b} 1 / j d \gamma u, u \in N(A D) \oplus R\left(\pi P A^{t}\right),
$$

and denote by $E$ the continuous extension of $E_{0}$ to $H_{T}$.

Lemma 3. $E$ is the orthogonal projection of $H_{T}$ onto the $H_{T}$ closure of $R\left(\pi P A^{t}\right)$.

Proof. Clearly $E \in L\left(H_{T}, H_{T}\right)$ and is self-adjoint. By Lemma 2, $E$ is fixed on the $H_{T}$ closure of $R\left(\pi P A^{t}\right)$. Since $E$ is zero on the orthogonal complement of $R\left(\pi P A^{t}\right)$ the lemma follows.

Proof of Theorem 1. Using Lemma 3, suppose $v \in N(A D) \oplus R\left(\pi P A^{t}\right)$ and

$$
u=v-\lim _{a \rightarrow 0+} \int_{a}^{b} 1 / j d \gamma v=(I-E) v .
$$

Since $I-E$ is the orthogonal projection of $H_{T}$ onto $N(A D)$ it must be that $A D u=$ 0 .

Now suppose that $A D u=0$. Then by the definition of $\gamma$,

$$
\int_{a}^{b} 1 / j d \gamma u=0,0<a<b
$$

and hence $(I-E) u=u$. This is enough to establish that $\Gamma=I-E$.

Proof of Theorem 2. Suppose $z$ is as in the statement of Theorem 2. Then

$$
z(t)=e^{-t A P A^{t}} v_{0}+\int_{0}^{t} e^{-(t-j) A P A^{t}} A D u, t \geq 0,
$$


and so

$$
\begin{aligned}
& \pi P A^{t} z(t)= \pi P A^{t} e^{-t A P A^{t}} v_{0}+\int_{0}^{t} \pi P A^{t} e^{-(t-j) A P A^{t}} A D u \\
&=\pi P A^{t} e^{-t A P A^{t}} v_{0}+\int_{0}^{t} e^{-(t-j) \pi P A^{t} A P} \pi P A^{t} A P D u \\
& \quad=\pi P A^{t} e^{-t A P A^{t}} v_{0}+\left(I-e^{-t \pi P A^{t} A P}\right) D u .
\end{aligned}
$$

Hence

$$
\lim _{t \rightarrow \infty} \pi P A^{t} z(t)=\lim _{t \rightarrow \infty}\left(I-e^{-t \pi P A^{t} A D}\right) u
$$

with the limit equal to $u$ minus the orthogonal projection of $u$ onto $N(A D)$, i.e., onto the $H_{T}$ closure of $R\left(\pi P A^{t}\right)$, since

$$
\lim _{t \rightarrow \infty} \pi P A^{t} e^{-t A P A^{t}} v_{0}=0 .
$$

\section{Conclusion}

Theorem 1 gives that each member of the quotient $H_{T} / M$ ( $M$ denoting the $H_{T}$ closure of $R\left(\pi P A^{t}\right)$ ) contains one and only one solution $u$ to $A D u=0$. It may be noted that given a point $x$ of a coset of this quotient, our first version of steepest descent leads directly to the solution in this coset.

It has been an object of some effort to obtain results analogous to those of this note for nonlinear problems. In [3], Chapter 14, there are some results (under rather strong conditions) along this line.

\section{REFERENCES}

[1] R. A. Adams, Sobolev Spaces, Academic Press, vol. 65, 1975. MR 56:9247

[2] A. Beurling, Collected Works, Vol 2, Birkhauser, 1989. MR 92k:01046b

[3] J. W. Neuberger, Sobolev Gradients and Differential Equations, Springer Lecture Notes in Mathematics \#1670 (1997). CMP 98:13

[4] F. Riesz and B. Sz-Nagy, Functional Analysis, Ungar, 1955. MR 91g:00002

[5] K. R. Payne, Boundary Geometry and Location of Singularities for Solutions to the Dirichlet Problem for Tricomi Type Equations, Houston J. Math. 23 (1997), 1-23.

[6] J. von Neumann, Functional Operators II, Annls. Math. Stud. 22, 1950. MR 11:599e

Department of Mathematics, University of North Texas, Denton, Texas 76203

E-mail address: jwn@unt.edu 